\title{
Proposal of a new Maximum Lifetime Communication Model of Wireless Sensor Network
}

\author{
https://doi.org/10.3991/ijoe.v12i10.6202 \\ Jinhui Lei and Xiyan Tian \\ Henan Institute of Science and Technology, Henan Xinxiang, China
}

\begin{abstract}
One new maximum lifetime communication model of wireless sensor network is proposed in this thesis and the model is based on conditions of base station with multiple sources and multiple links and the data generated from the source node can be transmitted to several base stations through multiple links. In this chapter, energy limitation and data stream conservation principles of a wireless sensor network are used to establish a linear planning model, and attaining the maximum lifetime of the network is the optimal objective to establish a model with multiple restrictions restricted by energy and bandwidth. The basic element influencing the lifetime of a wireless sensor network is the data forwarding rate. The lifetime of nodes will become longer with the decrease of node data. In this thesis, the data size of network nodes is taken as suboptimization objective and the distributed heuristic algorithm will be used to solve the linear planning model. In the end, a simulation experiment is conducted to verify yhe performance of the communication model and indicate that the proposed model can effectively increase network lifetime.
\end{abstract}

Index Terms-maximum lifetime, communication model, wireless sensor network.

\section{INTRODUCTION}

As shown in Fig. 1, the wireless sensor network consists of a sensor node, sink and manager node. A large number of sensor nodes are randomly distributed inside or near the monitoring area after being dripped and all nodes constitute the network through self-organization. The sensor node will monitor objects and data monitored in the initial processing will be transmitted by a multi-strip relay-operated method according to specific routing protocol. In the transmitting process, monitored data will be processed by several nodes and then they are routed to the aggregation nodes[1]. Then the data will be transmitted to manager nodes through a satellite, internet and mobile communication network. The end user will carry out configuration and management on the sensor network, issue a monitoring task and collect monitoring data.

In the multiple base station network of a wireless sensor, the network lifetime is increased to some degree when compared with the base station network. The network forms multiple groups in multiple base stations among which there are several similar route models to establish LEACH algorithm so as to form several clusters. The base station will be used to substitute cluster head in LEACH algorithm and every base station maintains surrounding node communication links. Furthermore, a hierarchal treeform communication structure CWSTP [2-3] is established for every base station and surrounding nodes in the network and each node only needs to communicate with the nearest base station, which can relieve data transmission pressure of the surrounding nodes in the base station, thus increasing network lifetime. As for nodes[45], one communication algorithm will be selected from multiple base stations, which is relatively similar to any cast communication technology in the TCP/IP protocol. Tony proposes one algorithm (MD-MLR) to realize maximum lifetime and minimum delay of wireless sensor network by any cast technology, and it mainly realizes the maximum lifetime of the network in data delay restrictions [6]. However, the communication model with multiple base stations is still insufficient in relieving communication pressure of surrounding nodes of the base station. In fact, the network is divided into several task areas according to distribution conditions of the base station and every base station is responsible for information collection conditions in one task area. To a certain degree, it is the collection of several models of a base station, which only shortens the node scale of base station maintenance in a single base station model.

One new maximum lifetime communication model of wireless sensor network is proposed in this thesis, and the model is based on conditions of a base station with multiple sources and multiple links. The data generated from the source node can be transmitted to several base stations through multiple links[7]. In this chapter, energy limitation and data stream conservation principles of a wireless sensor network are used to establish a linear planning model and maximum lifetime of network is the optimum objective to establish a model with multiple restrictions restricted by energy and bandwidth. The basic element influencing the lifetime of a wireless sensor network is the data forwarding rate[8]. The lifetime of nodes will become longer with the decrease of node data. In this thesis, the data size of network nodes is taken as sub-optimization objective and the distributed heuristic algorithm will be used to resolve the linear planning model. In the end, a simulation experiment is conducted to verify the performance of the communication model and indicate that the proposed model can effectively increase network lifetime, as shown in Figure 1. 


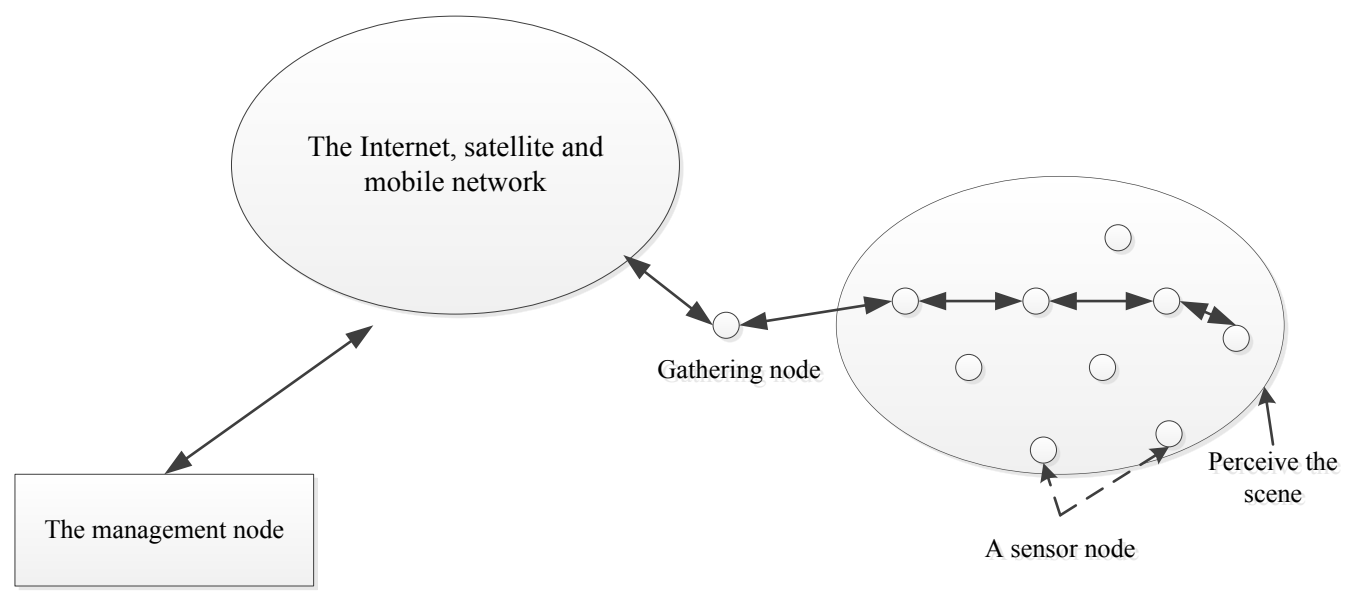

Figure 1. The architecture of wireless sensor network

\section{MLCM SYSTEM MODEL}

\section{A. Network model}

The wireless sensor network consists of a large number of sensor nodes which are densely distributed in a monitoring area for operation without engineering treatment or propositioning. Therefore, necessary sensor nodes will have self-organization characteristics. At the same time, multiple routes are necessary for transmission of data for the sensor nodes are embedded systems and the processing capacity, storage capacity and communication capacity are relatively weak. Additionally, apart from local information collection and data processing, nodes must store, manage and integrate data transmitted by other nodes. Furthermore, they must coordinate with other nodes and transmit original data to an aggregation node after processing[9-10]. As the interface of the sensor network and external network, the aggregation node can realize the communication between manager nodes and the wireless sensor network through network protocol. It will forward data collected from the sensor nodes to the external network and issue commands from the manager node to the sensor network at the same time, as shown in Figure 2.

The wireless sensor network can be abstracted as one undirected graph among $G(V, A)$ where $\mathrm{V}$ represents the set of sensor nodes and base stations in the network and $\mathrm{A}$ represents the set links. In the case that the set of sensor nodes in network is $\mathrm{S}$, the node number is $\mathrm{n}$, the set of base station is $\mathrm{s}$ and the number of base stations is $\mathrm{m}$, then $\mathrm{V}=\mathrm{S} \cup \bar{S}$. In the case that the set of adjacent node of $V_{i} \in V$ is $\mathrm{Si}$, then $V_{i}$ can have direct communication in any nodes inside $\mathrm{Si}$. The communication set of node $V_{i}$ is $\mathrm{X}$, $X \subseteq S_{i}$, and $\mathrm{X}$ consists of nodes between node $V_{i}$ and base stations and the communication link of node $\mathrm{Vi}$ is $\left(V_{i}, \mathrm{X}\right)$. The link set can be expressed as: $A=\left\{\left(V_{i}, \mathrm{X}\right) \mid V_{i} \in V, X \subseteq S_{i}\right\}$. The source node $\mathrm{V}_{\mathrm{k}}$ will perceive and generate data which will finally arrive at the base station node Sd through one or several frontier sets $\left(V_{i}, V_{j}\right)$ provided that the data size surpasses the frontier set $\left(V_{i}, V_{j}\right)$ in unit time in that process is $f_{V_{i}}^{s_{i}}, V_{j}$. In the case that the primary energy of all sensor nodes is $\mathrm{E}$, then the energy of node $\mathrm{V}_{\mathrm{i}}$ is $\mathrm{E}_{\mathrm{i}}$.

\section{B. Fundamental assumption}

The assumption is that the sensor network is of the following characteristics:

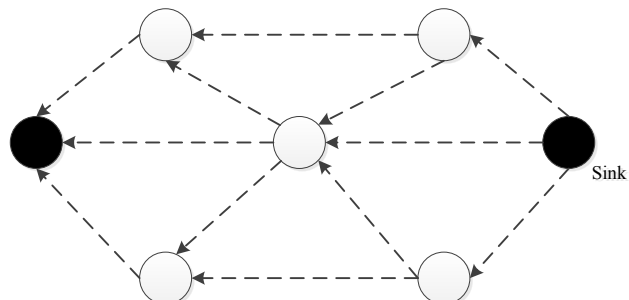

(a) The interest spread

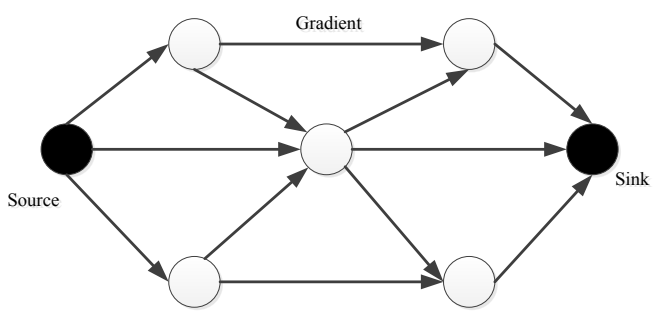

(b) Gradient based

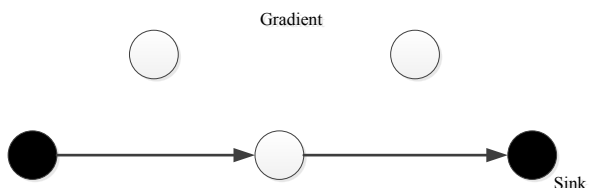

(c) Path to enhance

Figure 2. Network type

(1) The nodes in the network are uniformly distributed and the sensor nodes and the base station are stationary.

(2) All sensor nodes have the same initial energy and once the energy of a certain node is used up, the node will stop working.

(3) All nodes can transmit and receive data and partial nodes selected to periodically induce the data are called source nodes[11].

(4) All nodes have the same maximum transmission range $d_{\max }$ and the nodes with a distance from the base station is more than $d_{\max }$ the data is transmitted to the base station through the multi-hop routing mode.

(5) Networks are interconnected initially. 


\section{Energy consumption model}

The energy consumption in the node of the wireless sensor network includes three parts, which are respectively the processor module, sensor module and wireless communication module, of which the wireless communication consumes the most energy of the sensor node. The energy consumed by the wireless communication module in the sensor node is researched in this thesis, and the algorithm adopts the energy model used in Literature [12-13].

Each sensor node $V_{i} \in S$ continuously or periodically reports the data collected to the base station and the energy consumption of $\mathrm{V}_{\mathrm{i}}$ transmitting unit data to the neighbor node is:

$$
e_{i j}=\alpha+\beta \cdot \operatorname{Rang}_{i}^{m}
$$

Assuming the coordinates of the node and communication object $\mathrm{K}$ respectively are $\left(X_{i}, Y_{i}\right)$ and $\left(X_{j}, Y_{j}\right)$, then the communication radius is:

$$
g_{i}=\sum_{V_{i}, V_{j} \in V, V_{j} \subseteq X} f_{V_{i} V_{j}}^{V_{i} \bar{S}_{d}}
$$

In the case that the node does not have the positioning function and the node transmitting power is nonadjustable, the node generally broadcasts to the surrounding nodes with a fixed transmitting radius. Assuming the data generation rate of node $V_{i}$ is $g_{i}$, then:

$$
e_{t}(i, j)=e_{i j} \cdot \sum_{V_{i}, V_{j} \in V, V_{j} \subseteq X} f_{V_{i} V_{j}}^{V_{V} \bar{S}_{d}}
$$

Assuming the link bandwidth is $\mathrm{R}$ and it is allowed that the maximum data transmission amount of node $V_{i}$ and the adjacent node $\mathrm{V}_{\mathrm{j}}$ in unit time is $\mathrm{R}_{\mathrm{ij}}$, and then it is obvious that $f_{V_{i}, V_{k}}^{V, s} \leq R$. The energy consumption of communication between node $\mathrm{V}_{\mathrm{i}}$ and the adjacent node $\mathrm{V}_{\mathrm{j}}$ is $e(i, j)$.

$$
e_{r}(i)=\rho \cdot \sum_{V_{i}, V_{j} \in V, V_{j} \subseteq X} f_{V_{i} V_{j}}^{V_{i} \bar{s}_{d}}
$$

The energy consumption of node $\mathrm{Vi}$ receiving the data of adjacent node $\mathrm{V}_{\mathrm{j}}$ is $e_{r}(i)$ and $e_{r}(i)$ is unrelated to the distance between them, where, $\rho$ is the fixed constant. Other possible energy consumption of the node include $e_{g}$ representing the energy consumption of the node generating unit data, $e_{i d l e}$ the energy consumption of node in an idle state and $\mathrm{e}_{\text {listen }}$ the energy consumption of node continuously listening to the network state so as to promptly awaken the node. These three sources of energy consumptions are the same for all nodes and the energy consumption value is basically fixed, and given $e_{\text {other }}=e_{g}+e_{\text {idle }}+e_{\text {listen }}$, the energy consumption of the node $\mathrm{V}_{\mathrm{j}}$ within unit time is

$$
e(i, j)=e_{i j} \cdot \sum_{V_{i}, V_{j} \in V_{,} \subseteq X} f_{V_{i} V_{j}}^{V_{i} \bar{S}_{d}}+\rho \cdot \sum_{V_{i}, V_{j} \in V_{,} \subseteq X} f_{V_{i} V_{j}}^{V_{i} \bar{S}_{d}}+e_{\text {other }}
$$

\section{Communication model}

The maximum lifetime communication model of the wireless sensor network proposed in this thesis is based on a multi-source, multi-link and multi-base station condition. The data generated by the source node can be transmitted to different base station nodes through different links[14].
The source node generates data, the intermediate node provides data forwarding service, the data continuously converges to the base station node from the edge node in the network and the source node induces the data generated and finally arrives at the base station node through single-hop or multi-hop forwarding. Data generated by nodes of the same source may be routed to different base station nodes through different links and it is of very strong dependency[15]. Different source nodes may conduct data fusion processing in the process of data forwarding. On account of the base station node requiring data from the same source for the fusion processing, multiple base station nodes form a tree structure and the root node is connected with the virtual link through each base station, of which one base station node is taken as the root node and it conducts redundancy removal to compress all data and then sends it to the terminal receiver.

\section{MLCM COMMUNICATION MODEL}

\section{A. Maximum lifetime model}

The wireless sensor network is the energy restriction network and the energy consumption of the node will not exceed the residual energy. Based on Equation (7), when the data forwarding rate of the node is $f$, the lifetime of a node $\mathrm{V}_{\mathrm{i}}$ is

$$
\begin{aligned}
& T_{i}(f)=\frac{E_{i}}{p_{r}(i)+p_{t}(i, j)+e_{\text {other }}} \\
& =\frac{E_{i}}{e_{i j} \cdot \sum_{V_{i}, V_{j} \in V, V_{j} \subseteq X} f_{V_{i} V_{j}}^{V_{i} \bar{S}_{d}}+\rho \cdot \sum_{V_{i}, V_{j} \in V, V_{j} \subseteq X} f_{V_{i} V_{j}}^{V_{i} \bar{S}_{d}}+e_{\text {other }}}
\end{aligned}
$$

The nodes of the wireless sensor network under working conditions based on the effect of node on the data stream can be divided into source node and intermediate node. The source node induces, generates and receives data and then forwards the data to the next adjacent node. The intermediate node does not generate data, but only receives data and then forwards to the next adjacent node. The data stream conditions of the two nodes are:

$$
\sum_{V_{i}, V_{j} \in V, V_{j} \subseteq X} f_{V_{i} V_{j}}^{V_{i} \bar{S}_{d}}+\lambda_{i} g_{i}=\sum_{V_{i}, V_{j} \in V, V_{j} \subseteq X} f_{V_{i} V_{j}}^{V_{i} \bar{S}_{d}}
$$

Where,

$$
\lambda_{i}=\left\{\begin{array}{l}
1, V_{i} \neq \bar{S}_{d} \\
-1, V_{i}=\bar{S}_{d}
\end{array}\right.
$$

Based on Equation (8), the lifetime model of network node is:

$$
T_{i}(f)=\frac{E_{i}}{e_{i j}\left(\sum_{V_{i}, V_{j} \in, V_{j} \subseteq X} f_{V_{i} V_{j}}^{V_{i} \bar{s}_{d}}+\lambda_{i} g_{i}\right)+\rho \cdot \sum_{V_{i}, V_{j} \in V_{,} \subseteq X} f_{V_{i} V_{j}}^{V_{i} \bar{S}_{d}}+e_{\text {other }}}
$$

The lifetime $T_{s y s}(f)$ of the network at a certain data forwarding rate $\mathrm{f}$ is the minimum lifetime of all nodes.

$$
T_{s y s}(f)=\min _{i \in S} T_{i}(f)
$$

The objective of the energy-efficient network communication model is to maximize the minimum lifetime of the node and prolong the network lifetime as much as possible to make the energy consumption of a single node balanced with the whole network[16]. The purpose of research on the maximum lifetime communication model of wireless sensor network is to 
maximize the network lifetime. The maximum lifetime communication model established in this chapter is:

$$
\begin{aligned}
& \max \text { imize } \min _{i \in S} T_{i}(f) \\
& \begin{array}{c}
\sum_{V_{i}, V_{j} \in V, V_{j} \subseteq X} f_{V_{i} V_{j}}^{V_{V_{d}} \bar{S}_{d}}+\lambda_{i} g_{i}= \\
\sum_{V_{i}, V_{j} \in V, V_{j} \subseteq X} f_{V_{i} V_{j}}^{V_{i} \bar{S}_{d}}, \forall V_{i}, V_{j} \in V, \forall \bar{S}_{d} \in \bar{S}
\end{array} \\
& T_{i}(f)\left(e_{i j} \cdot \sum_{V_{i}, V_{j} \in V, V_{j} \subseteq X} f_{V_{i} V_{j}}^{V_{i} \bar{S}_{d}}+\lambda_{i} g_{i}+\right. \\
& \left.\left.\rho \cdot \sum_{V_{i}, V_{j} \in V, V_{j} \subseteq X} f_{V_{i} V_{j}}^{V_{i} \bar{S}_{d}}+e_{\text {other }}\right) \leq E_{i}\right) \\
& \sum_{S_{d} \in S} f_{V_{i} V_{j}}^{V_{i} \bar{S}_{d}} \leq R_{i j}, \forall V_{i}, V_{j} \in X, X \subseteq S_{i} \\
& f_{V_{i} V_{j}}^{V_{i} \bar{S}_{d}} \geq 0, \lambda_{i}=0 \text { orl }, g_{i} \geq 0, \forall V_{i}, V_{j} \in X, X \subseteq S_{i}
\end{aligned}
$$

The Condition 1 in Equation (12) shows that the quantity of data transmitted by node $\mathrm{Vi}$ to the adjacent node is equal to the sum of data generated by the data induction and the quantity of data received from the adjacent node, and the data loss in transmission is not considered in this chapter. Condition 2 shows that the total energy consumption of node I within time of $T_{\text {sys }}(f)$ will not exceed the set value $E_{i}$ of the node energy. Condition 3 shows that the communication rate between the node and the adjacent node is less than the network bandwidth. The condition of the node not participating in the network communication is not considered in this thesis, such as the conditions of the dormant node, a node without a monitoring area and the node that has accidentally gone dormant in deployment[17].

\section{B. Data stream model}

The base station set $\bar{S}=\left\{\bar{S}_{1} \bar{S}_{2, \ldots}, \bar{S}_{\mathrm{m}}\right\}$ can form $2 \mathrm{~m}-1$ nonvoid subsets. In the case of assuming $\mathrm{m}=2$ and $\bar{S}=\left\{\bar{S}_{1}, \bar{S}_{2}\right\}$, the subsets possibly formed are $\left\{\bar{S}_{1}\right\},\left\{\bar{S}_{2}\right\}$ and $\left\{\bar{S}_{1} \bar{S}_{2}\right\}$. In each process of the source node transmitting data, the data will be transmitted to a certain subset of the base station. In the adjacent communication link $\left\{V_{i}, X\right\}$ of the source node, the set $\mathrm{X}$ is not void with $X \in S_{i}$ and $X=\left\{X_{1}, X_{2}, \ldots, X_{k}\right\}$; the data of mode $\mathrm{Vi}$ is routed to one base station as the least through $\mathrm{k}$ links and assuming the node $\mathrm{Vi}$ passes through the adjacent node X1 and transmits to the subset $\mathrm{P} 1$ of the base station, and transmits the subset P1 of the base station through node $\mathrm{X} 2$; that is, the set transmitted to the base station subset through the adjacent communication link set $\mathrm{X}$ is

$$
P=\left\{P_{1}, P_{2}, \ldots, P_{k}\right\} \text { and } P \in \bar{S}
$$

The subset of node Vi transmitted to the base station through the adjacent node $\mathrm{V}_{\mathrm{j}}$ is $\mathrm{P}_{\mathrm{k}}$ with $P_{k} \notin \Phi$ and $P_{k}=\left\{P_{1}, P_{2}, \ldots, P_{k}\right\}$; assuming that the data forwarded by node $\mathrm{Vi}$ through the adjacent node $\mathrm{V}_{\mathrm{j}}$ is received by $\mathrm{Pk}$ base station subset at the average data rate of $F_{V_{V} V_{i}}^{V_{1}}$ and total amount of data received by $\mathrm{P}_{\mathrm{k}}$ base station is $F_{V_{i} V_{j}}^{V_{i}}$ $\left\{P_{1}, P_{2}, \ldots, P_{k}\right\}$.

In the communication model, $F_{V_{i} V_{i}}^{V_{s}, S_{d}}$ represents the quantity of data passing through the edge set $\left(V_{i}, V_{j}\right)$ within a unit of time and based on the data stream model, the relation between $F_{V_{i}, V_{j}}^{V_{s}, S_{d}}$ and the average data rate $F_{V_{i} V_{i}}^{V_{i}}$ arriving at the base station set is:
$\sum_{V_{j} \in X, X \subseteq S i} f_{V_{i} V_{j}}^{V_{S^{\prime}} \bar{S}_{d}}=F_{V_{i} V_{j}}^{V_{i}}\left(\left\{\overline{\mathrm{S}}_{1}\right\}\right)+F_{V_{i} V_{j}}^{V_{i}}\left(\left\{\overline{\mathrm{S}}_{2}\right\}\right)+F_{V_{i} V_{j}}^{V_{i}}\left(\left\{\bar{S}_{1}, \overline{\mathrm{S}}_{2}\right\}\right)$

Assuming the subset collections formed by $F_{V_{s} V_{j}}^{V_{s}, S_{a}}$ reaches $\bar{S}=\left\{\bar{S}_{1} \bar{S}_{2}\right\}$ are $\left\{\bar{S}_{1}\right\}$ and $\left\{\bar{S}_{2}\right\}$.

$f_{V_{i} V_{j}}^{V_{i} \bar{S}_{d}}=F_{V_{i} V_{j}}^{V_{i}}\left(\left\{\overline{\mathrm{S}}_{1}\right\}\right)+F_{V_{i} V_{j}}^{V_{i}}\left(\left\{\overline{\mathrm{S}}_{2}\right\}\right)$

In that case that node $\mathrm{V}_{\mathrm{i}}$ arrives at the collection of all base stations through the adjacent node collection $\mathrm{V}_{\mathrm{j}}$; that is, all subset collections are formed by $\bar{S}=\left\{\bar{S}_{1,} \bar{S}_{2}\right\}$

$$
\sum_{V_{j} \in X, X \subseteq S i} f_{V_{i} V_{j}}^{V_{i} \bar{S}_{d}}=F_{V_{i} V_{j}}^{V_{i}}\left(\left\{\overline{\mathrm{S}}_{1}\right\}\right)+F_{V_{i} V_{j}}^{V_{i}}\left(\left\{\overline{\mathrm{S}}_{2}\right\}\right)+F_{V_{i} V_{j}}^{V_{i}}\left(\left\{\bar{S}_{1}, \overline{\mathrm{S}}_{2}\right\}\right)
$$

It is assumed in this thesis that the subset collection of data transmitted by node $\mathrm{V}_{\mathrm{i}}$ arrives at the base station is $\Omega$ with $\Omega \subseteq p$ and $\Omega=\left\{P_{1}, P_{2}, \ldots, P_{k}\right\} . \phi_{V i}(\Omega)$ Represents the total rate of data transmitted by node $\mathrm{V}_{\mathrm{i}}$ to $\Omega$ within a unit of time.

$\varphi_{\mathrm{Vi}}(\Omega)=\sum_{P_{k} \subseteq P, S_{d} \in P_{k}} F_{V_{i} V_{j}}^{V_{i}}\left(P_{k}\right)=\sum_{V_{j} \in X, X \subseteq S_{i}} \sum_{P_{k} \subseteq P} F_{V_{i} V_{j}}^{V_{i}}\left(P_{1}+P_{2}+\mathrm{L}+P_{k}\right)$

The data transmitted by node $\mathrm{V}_{\mathrm{i}}$ comes from the sum of data received and the data generated by its induction, and the data received by the node $\mathrm{Vi}$ from the adjacent node is $\sum_{V_{i}, V_{j} \in V, Y_{i} \subseteq X} f_{V_{i}, V_{j}}^{V_{g}, S_{d}}$ and $\phi_{V i}(\Omega)$ represents the total rate of data received by a node within a unit of time.

$\varphi_{i}(\Omega)=\sum_{h \in X} \sum_{P_{k} \subseteq P} F_{V_{i} V_{j}}^{V_{i}}(P k)=\sum_{V_{j} \in\left\{, x \subseteq S_{i}\right.} \sum_{P_{k} \leq P} F_{V_{i} V_{j}}^{V_{i}}\left(P_{1}+P_{2}+\mathrm{L}+P_{k}\right)$

In this chapter, the value of $\phi_{V i}(\Omega)$ generally is more than that of $\phi_{V i}(\Omega)$; given $\Psi_{i}(\Omega)=\phi(\Omega)-\varphi(\Omega)$ and in the case of $\Psi_{i}(\Omega)>0$. In addition to forwarding data within a unit of time, the node $\mathrm{Vi}$ also induces and generates data, and $\Psi_{i}(\Omega)=0$ shows that the node $\mathrm{V}_{\mathrm{i}}$ only forwards data and $\Psi_{i}(\Omega)<0$ shows that there is data loss in node Vi or it conducts redundancy processing and data compression for the data[18]. It can be determined from Equation (7) that when the data rate in the node decreases, the energy consumption of the node will also decrease, and when there is no data passing the node, it is considered that the node only needs to maintain relatively low energy consumption, as shown in Figure 3.

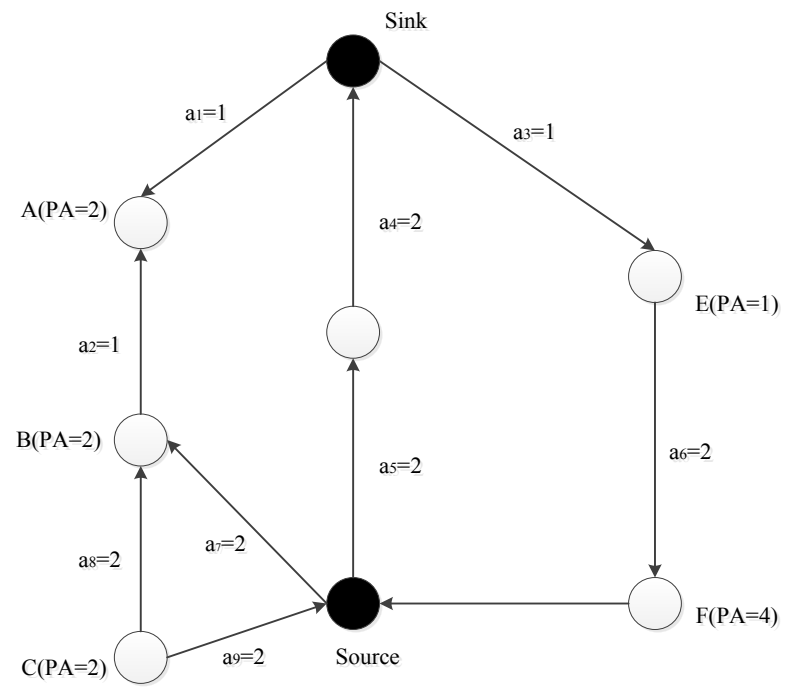

Figure 3. Energy routing protocol 


\section{Systematic mathematic model}

In this chapter, a multi-constraint linear programming model is proposed in order to resolve the maximum lifetime communication model of the wireless sensor network, with network lifetime being its optimal objective. A distributed algorithm is a typical algorithm adopted to solve linear programming model. In order to resolve the maximum lifetime model of a network with adoption of distributive algorithm, substitution variable $\mathrm{q}$ is introduced, and set $q=1 / T_{\text {sys }}(f)$. The solution to the maximum lifetime of a wireless sensor network becomes the solution to minimization of $\mathrm{q}$, and such a model can be transformed into an equivalent model.

maximize $q$

$$
\begin{aligned}
& \sum_{V_{i}, V_{j} \in V, V_{j} \subseteq X} f_{V_{i} V_{j}}^{V_{i} \bar{S}_{d}}+\lambda_{i} g_{i} \\
& \text { subject to } \\
& =\sum_{V_{i}, V_{j} \in V, V_{j} \subseteq X} f_{V_{i} V_{j}}^{V_{i} \bar{S}_{d}} \\
& e_{i j} \cdot \sum_{V_{i}, V_{j} \in V, V_{j} \subseteq X} f_{V_{i} V_{j}}^{V_{S} \bar{S}_{d}}+\lambda_{i} g_{i}+ \\
& \rho \cdot \sum_{V_{i}, V_{j} \in V, V_{j} \subseteq X} f_{V_{i} V_{j}}^{V_{i} \bar{S}_{d}}+e_{\text {other }} \leq q E_{i}, \forall V_{i}, V_{j} \in V, \forall \bar{S}_{d} \in \bar{S} \\
& \sum_{S_{d} \in S} f_{V_{i} V_{j}}^{V_{i} \bar{S}_{d}} \leq R_{i j}, \forall V_{i}, V_{j} \in X, X \subseteq S_{i} \\
& f_{V_{i} V_{j}}^{V_{i} \bar{S}_{d}} \geq 0, \lambda_{i}=0 \text { orl }, g_{i} \geq 0, \forall V_{i}, V_{j} \in X, X \subseteq S_{i}
\end{aligned}
$$

The objective function $\mathrm{q}$ is technically not a convex function and the dual function is non-differentiable. To solve this problem, the substitution scheme proposed in literature [19] is adopted, where $\mathrm{q}$ is substituted with $\mathrm{q}^{2}$, and such substitution has no influence on the solution of maximum value of lifetime T. Meanwhile, in this chapter, a differential convex secondary coordination item $\phi(x)$ is introduced based on the characteristics of a wireless sensor network. By setting as $\phi(x)=\Psi(\Omega)$ the KKT condition is satisfied. In order to make $\phi(x)$ become differential or technically a convex function, substitute $\Psi_{i}(\Omega)$ with $\sigma^{2} \Psi_{\mathrm{t}}^{2}(\Omega)$, where $\sigma$ is a small positive real number; the linear programming model will be:

$$
\begin{aligned}
& \text { maximize } q^{2}+\left(\varphi_{i}(\Omega)\right) \\
& \sum_{\text {subject to } \sum_{i}, V_{j} \in V, V_{j} \subseteq X} f_{V_{i} V_{j}}^{V_{S} \bar{S}_{d}}+\lambda_{i} g_{i} \\
& \text { subject to } \quad=\sum_{V_{i}, V_{j} \in V, V_{j} \subseteq X} f_{V_{i} V_{j}}^{V_{i} \bar{S}_{d}} \\
& e_{i j} \cdot \sum_{V_{i}, V_{j} \in V, V_{j} \subseteq X} f_{V_{i} V_{j}}^{V_{i} \bar{S}_{d}}+\lambda_{i} g_{i}+ \\
& \rho \cdot \sum_{V_{i}, V_{j} \in r, V_{j} \subseteq X} f_{V_{i} V_{j}}^{V_{i} \bar{S}_{d}}+e_{\text {other }} \leq q E_{i}, \forall V_{i}, V_{j} \in V, \forall \bar{S}_{d} \in \bar{S} \\
& \sum_{S_{d} \in S} f_{V_{i} V_{j}}^{V_{i} \bar{S}_{d}} \leq R_{i j}, \forall V_{i}, V_{j} \in X, X \subseteq S_{i} \\
& \varphi_{i}(\Omega)=\sum_{V_{j} \in X, X \subseteq S_{i}} \sum_{P_{k} \subseteq P} F_{V_{i} V_{j}}^{V_{i}}\left(P_{1}+P_{2}+\mathrm{L}+P_{k}\right) \\
& \Psi_{i}(\Omega)=\phi_{i}(\Omega)-\varphi_{i}(\Omega)
\end{aligned}
$$

$f_{V_{i} V_{j}}^{V_{i} \bar{S}_{d}} \geq 0, \lambda_{i}=0$ or $1, g_{i} \geq 0, \forall V_{i}, V_{j} \in X, X \subseteq S_{i}$

Equation (19) is the linear programming model described in the optimization theory, which can be resolved with the adoption of the distributed projected gradient algorithm. The approximate upper limit value of the maximum network lifetime can be solved through iterative computation.

\section{SIMULATION AND PERFORMANCE ANALYSIS ON THE ALGORITHM}

In order to verify performances of the entire model, simulation has been conducted in NS-2 environment, and the simulation result was applied based on WSTP and MD-MLR to realize random generation in NS-2 environment of 2-6 sink nodes and 30-100 common sensor nodes which are randomly distributed within a planar area measuring $80 \mathrm{X} 80 \mathrm{M} 2$. All these nodes are equipped with positioning function and their transmission power is adjustable. $\mathrm{R}$, radius of maximum transmission range, is 15 ; data generation rate $\mathrm{g}_{\mathrm{j}}=\mathrm{lkbit} / \mathrm{s}$; data package size is 512Bytes; metadata size is 25Bytes; primary energy transmission unit and data energy consumption factor of sensor node are subject to comparison. $\sigma=50 \mathrm{~nJ} / \mathrm{b}$, $\beta=0.0013 \mathrm{pJ} / \mathrm{b} / \mathrm{m} 4$, and data energy consumption factor of the receiving part $\rho=50 \mathrm{~nJ} / \mathrm{b}$; path power consumption model parameter $\mathrm{m}=4$; correction factor of secondary optimization model $\sigma=0.01$.

Figure 4 shows the relationship between total energy consumption of the network and the number of nodes. The number of base station is respectively set as 2, 3, 4, 5 and 6. As the number of network nodes increases, node intensity increases. Meanwhile, the number of source nodes increases proportionally. In fact, when the number of nodes doubles, the data size generated in the network doubles correspondingly. It is obvious that as node intensity increases, the increased energy consumption will be less than doubled. However, the total energy consumption of the network will rise. As the number of base station increases, the total energy consumption rate of the network reduces gradually. With number of nodes being the same, the more the base station there is, the closer is the link formed by source node data to the base station, resulting in less energy consumption. When the number of base station increases, the reduction of average energy consumption of the network caused by the newly accessed base station becomes less. In such a case, as $n=30$, when the number of base station expressed as $m$, increases from 2 to 4 , energy consumption will be reduced about $32 \%$. In other words, when the number of base station doubles, reduction in energy consumption will not exceed $50 \%$. When $\mathrm{m}$ is increased to 6 , the reduction in energy consumption is such that it is about $48 \%$ of that when $\mathrm{m}=2$, and $16 \%$ further less than when $\mathrm{m}=4$. The influence of the number of base stations on the total network energy consumption reduces gradually, while the total network energy consumption shows a declining trend.

Figure 5 shows the comparison between the proposed algorithm MLCM and WSTP and MD-MLR algorithm in terms of maximum network lifetime when there are three base stations in the network. It can be observed from Figure 5 that the algorithm proposed in this chapter is superior to the other two models in terms of network lifetime. According to the MLCM algorithm, data 
generated at the source node can be transmitted, in the process of data routing, via the optimal link among many links, or otherwise transmitted via all these links, and the number of such links may increase when data is transmitted from the source node. The MLCM model tries to cause all nodes in the network to participate in the data transmission in order to make the network energy evenly consumed by more nodes, although nodes participating in the transmission may not be located within the task section of the WSTP algorithm. For the WSTP algorithm, the network is divided into sections based on the number of base stations, and each section is under the charge of a certain base station. It is in fact a convergence of a singlebase-station network, which is very close to the shortest path model of common with a single base station in regards to energy consumption. In this case, energy consumption is likely to take place at a certain link, making nodes on such link die excessively quickly, which results in a short link lifetime. The MD-MLR algorithm is an event-driven routing model and is the routing algorithm based on minimum network delay and maximum network lifetime. According to this algorithm, route discovery will be re-conducted prior to each routing. The algorithm seeks to improve the efficiency of transmission to the base station and reduce delay. However, the path of data transmission fails to realize the optimal minimum energy consumption path on general network energy consumption, making the optimization unsatisfactory.

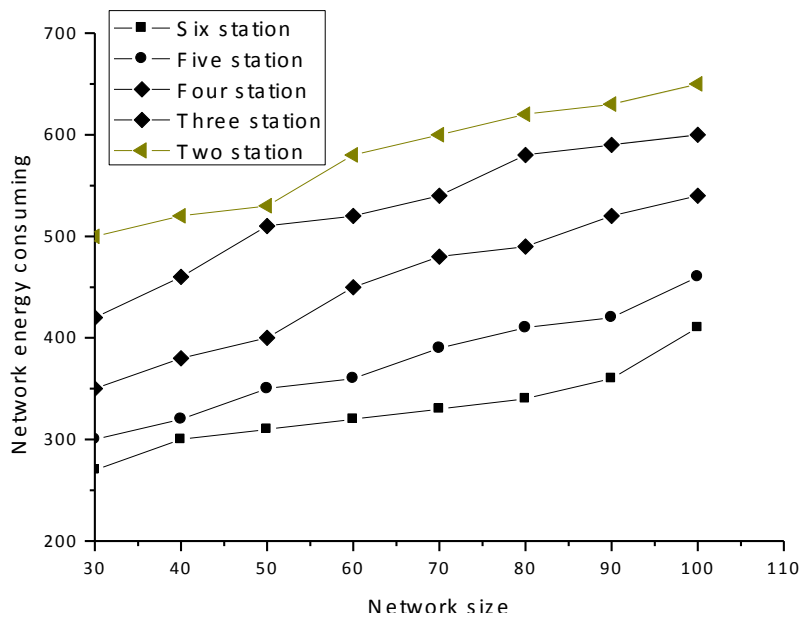

Figure 4. Network energy consumtion

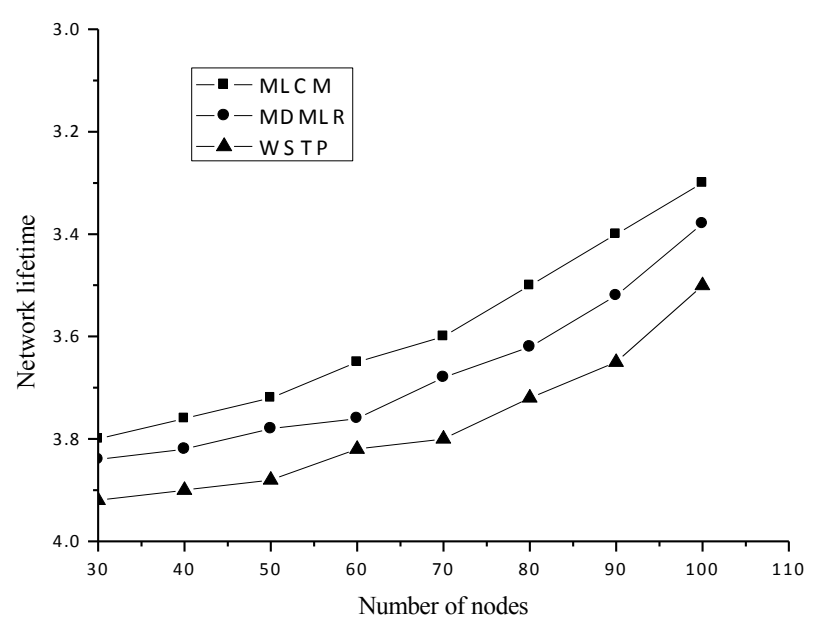

Figure 5. Comparison of network lifetime

\section{CONCLUSION}

In this thesis, research has been conducted on the maximum lifetime communication model of a multisource multi-link multi-base station of a wireless sensor network. Multiple base stations are often deployed in wireless sensor networks in order to prolong lifetime, reduce data delay and reinforce network robustness. In terms of wireless sensor network equipped with multiple base stations, data generated at the source node can be transmitted to a number of base stations via multiple links. In the process of data transmission, the primary objective is to realize maximum network lifetime, and the secondary objective is to reduce data size at the node; namely, to make data arrive at the base station via the minimum number of nodes in the network. A linear programming model which takes a maximum network lifetime as the optimal objective is established in order to cope with many constraints such as limited energy and bandwidth of a wireless sensor network. Such a linear programming model is resolved with the adoption of a distributed heuristic algorithm. Finally, performances of the communication model have been verified through a simulation experiment, which shows that the proposed model is effective in improving network lifetime.

\section{REFERENCES}

[1] L.Y. Min, J. X. Hua, "A protocol model for wireless sensor network," Proceedings - International Conference on Networks Security, Wireless Communications and Trusted Computing, vol. 2, pp. 588-591, April 2009.

[2] R.A. Radhika, "Routing in wireless sensor network using fuzzy based trust model," Proceedings - 2014 4th International Conference on Communication Systems and Network Technologies, vol.4, pp. 529-532, May 2014.

[3] N. Jan, K. Ryszard, N. Maciej, "Directed communication in Wireless Sensor Network based on Digital Terrain Model” 2009 2nd International Symposium on Logistics and Industrial Informatics, LINDI 2009, pp.231-236.

[4] H.Q. Long, G.Z. Guo, "Research on cloud trust model for malicious node detection in wireless sensor network," Tien Tzu Hsueh Pao/Acta Electronica Sinica, vol. 40, pp. 2232-2238, November 2012.

[5] K. Celalettin, "Energy and lifetime analysis of compressed Wireless Sensor Network communication," 2013 IEEE Sensors Applications Symposium, SAS 2013-Proceedings, pp. 7-10.

[6] K. Neha, "Mathematical model on the transmission of worms in wireless sensor network," Applied Mathematical Modelling, vol. 37, pp. 4103-4111, March 2013. http://dx.doi.org/10.1016/j.apm.2012.09.025

[7] C. Xiaojuan, D.K. Mieso, "Modelling the energy cost of a fully operational wireless sensor network," Telecommunication Systems, vol. 44, pp. 3-15, June 2010. http://dx.doi.org/10.1007/s11235-009-9228-z

[8] W. Ruchuan, S. Lijuan, "Study on communication model between heterogeneous operating systems for a wireless sensor network middleware," Chinese Journal of Electronics, vol. 16, pp. 543546, July 2007.

[9] X.S. Zhi, W.Q. Chen, "Attenuation model of antenna signal with barriers in wireless sensor network," Applied Mechanics and Materials, vol. 380-384, pp. 3908-3911, May 2013. http://dx.doi.org/10.4028/www.scientific.net/AMM.380-384.3908

[10] L.H. Guang, "Full interference model in wireless sensor network simulation," Proceedings of the 2009 6th International Symposium on Wireless Communication Systems, ISWCS2009, pp. 647-651.

[11] M. Michael, H. Sajid, "Investigating wireless sensor network lifetime using a realistic radio communication model," Proceedings-2008 International Conference on Multimedia and Ubiquitous Engineering, MUE 2008, pp. 433-437. 
[12] S. Aghaeine, "Modeling of earthquake prediction system on wireless sensor network via satellite communication," Proceedings of the International Astronautical Congress, vol. 5, pp. 3332-3336, April 2013.

[13] W. Yan, "Study on model and architecture of self-organization wireless sensor network, 2008 International Conference on Wireless Communications," Networking and Mobile Computing, WiCOM 2008, pp.306-312.

[14] H.J. Qiang, Z. Wei, Z. Mao, "An analytical model on unbalanced energy consumption in large scale wireless sensor network," Proceedings - 3rd International Conference on Measuring Technology and Mechatronics Automation, ICMTMA 2011, pp. 375-378.

[15] B. Suman, G. Chandan, "Fuzzy communication model for sensors in Wireless Sensor Network, Proceedings of the 2012 International Conference on Communications," Devices and Intelligent Systems, CODIS 2012, pp. 254-257.

[16] S. Amrita, S. Pinaki, "Highly resilient communication using affine planes for key predistribution and Reed Muller codes for connectivity in wireless sensor network," Communications in Computer and Information Science, vol. 162, pp. 83-94, August 2011. http://dx.doi.org/10.1007/978-3-642-21937-5 9

[17] C. X. Jun, W.M. Rong, "Research on self-adaptive wireless sensor network communication architecture based on ptolemy ii," Communications in Computer and Information Science, vol. 159 pp. 90-95, April 2011. http://dx.doi.org/10.1007/978-3-64222691-5 16

[18] L. Mariom, "Modelling and simulation approach for the performance evaluation of a wireless sensor network," Proceedings of the IASTED International Conference on Automation, ACIT-ICT 2010, pp. 140-145.

[19] C. Frank, S. Shyamala, "A clustered wireless sensor network model based on log - distance path loss," Proceedings of the 6th

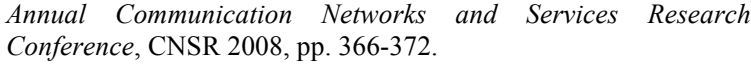

\section{AUTHORS}

Jinhui Lei received his bachelor's degree in Electronic Information Engineering from Zhengzhou University, Zhengzhou, Henan, China, in 2003, and the master's degree in Electronics and Communication Engineering from Zhengzhou University, Zhengzhou, Henan, China, in 2011. Now he is now a lecturer at School of Information Engineering, Henan Institute of Science and Technology, Xinxiang 453003, China. His current research interests include embedded systems, digital signal processing, image processing, pattern recognition. (ljh@hist.edu.cn).

Xiyan Tian received the B.E. in application of electronic technology education from Henan Normal University, Xinxiang, Henan, China, in 2002, and the M.S in communication and information system from Zhengzhou University, Zhengzhou, Henan, China, in 2014. Now, she is a lecturer at School of Mechanical and Electrical, Henan Institute of Science and Technology, Xinxiang 453003, China. Her research interests mainly focused on wireless communication, wireless sensor networks and speech signal processing. (tianxy2001@126.com).

Submitted 03 September 2016. Published as resubmitted by the authors 16 October 2016. 